\title{
Comparison of TI-RADS classification with FNAC for the Diagnosis of Thyroid Nodules
}

\author{
Naushaba Malik ${ }^{1}$, Maryam Rauf ${ }^{2}$, Ghazala Malik $^{3}$ \\ ${ }^{1}$ Head, Department of Radiology, PESSI Hospital, Islamabad, Pakistan \\ ${ }^{2}$ Consultant, Department of Radiology, PESSI Hospital, Islamabad, Pakistan \\ ${ }^{3}$ Radiologist, Pakistan Institute of Medical Sciences, Islamabad, Pakistan
}

\begin{abstract}
Background: Thyroid nodules are very common and its prevalence is largely dependent on the identification techniques. Recently high-resolution ultrasound thyroid imaging has paved the way for significant transformation in clinical approach to thyroid nodule. This study aimed to determine the efficacy of TI-RADS classification and its association with FNAC findings in thyroid lesions.

Material and Methods: This prospective study was carried out in the Department of Radiology of Islamabad Diagnostic Centre, Islamabad for a period of 6 months from $6^{\text {th }}$ January 2018 to $6^{\text {th }}$ July, 2018. All male and female patients presenting with thyroid nodules were selected for the study. Ultrasound neck was performed with high frequency linear probes. Ultrasonography findings were classified according to Thyroid Imaging Reporting and Data System (TI-RADS) classification, as defined by Horvath et al. Patients with TI-RADS II-V were scheduled for US-guided Fine Needle Aspiration (FNA). All the specimens were sent to pathology laboratory for cytology (FNAC).

Results: Total 123 patients of thyroid nodules were studied. Mean age of the patients was $45.78 \pm 13.11$ years, with a female predominance (73.2\%). A significant association was seen between TI-RADS classification of thyroid nodules and findings on cytology. Thyroid nodules with TI-RADS II, III and IVa classification showed benign cytological findings, while TI-RADS class $V$ had a significant association with malignant findings on cytology $(P=0.001)$.

Conclusions: TI-RADS classification is a reliable modality in differentiating benign thyroid nodules from malignant ones and circumvent the need for FNAC in every case with a thyroid nodule.
\end{abstract}

Key words: TI-RADS, Cytology, FNAC, Thyroid nodules

Authors' Contribution: Correspondence:

${ }^{1-3}$ Conception; Literature research; Naushaba Malik

manuscript design and drafting; Critical Email: sazinlondon@gmail.com

analysis and manuscript review; Data

analysis; Manuscript Editing.

Cite this article. Malik N, Rauf M, Malik G. Comparison of TI-RADS classification with FNAC

for the Diagnosis of Thyroid Nodules. J Islamabad Med Dental Coll.2020; 9(2): 129-133. Doi:

10.35787/jimdc.v9i2.485

\section{Introduction}

The thyroid gland (an essential endocrine gland) is present anterior to the trachea at the base of neck. ${ }^{1}$ Overall, thyroid nodules are quite prevalent, and most of these nodules are benign. A thyroid nodule is the development of a lump (solid or fluidfilled) in the substance of thyroid gland.
Ultrasonographically, thyroid gland lesions are distinguishable from the surrounding normal thyroid parenchyma. ${ }^{2}$ In case of thyroid malignancies, treatment of thyroid nodules is becoming a debatable subject. About $2-6 \%$ of thyroid nodules are easily detected by palpation, 
$19-68 \%$ are diagnosed with ultrasonography (USG), however $8-65 \%$ are discovered on autopsy. ${ }^{3,4}$ Among those detected through palpation or the USG, the incidence of carcinoma is about 5-15\%., In a population-based research, the malignancy rate among fine-needle aspiration (FNA) assessed non-palpable nodules was $8-12 \%$, and among thyroid nodules detected through palpation was $1.6 \%$. $^{3,5,6}$

Among endocrine glands, thyroid cancer is the most prevalent malignancy, accounting for $3.8 \%$ of all newly appearing cancer patients in the U.S and is the ninth most prevalent cancer, in general. ${ }^{1}$ The number of individuals diagnosed with thyroid malignancy has risen significantly over the last 30 years. ${ }^{2}$ Thyroid FNAC is the primary thyroid nodule diagnostic test. Thyroid FNAC is graded into six groups by the Bethesda system and also predicts the risk of carcinoma. ${ }^{7}$ USG-guided FNA has an important contribution in managing thyroid nodules because of its accuracy and safety. However, even with USG guidance, sampling errors or non-representative sampling can occur during FNA. ${ }^{8}$ Still it is regarded as a cost-effective method of investigation for thyroid nodules. ${ }^{9}$

Horvath et al proposed TI-RADS for ultrasonographic evaluation and classification of patients with thyroid nodule prior to FNAC/FNA biopsy to safeguard them from unwanted complications. ${ }^{10,11}$ Some studies found TI-RADS classification as a sensitive method for identifying patients with thyroid cancer, ${ }^{12,13}$ while others advocated the need of cancer prediction with more sensitive gold-standard technique of FNAB. ${ }^{13}$ This study was planned in the context of these controversies and unavailability of relevant data in local settings. The objective was to compare the effectiveness of TI-RADS classification with cytological confirmation in diagnosing thyroid nodules.

\section{Material and Methods}

This prospective study was performed at the Radiology Department of Islamabad Diagnostic Center, Islamabad Pakistan. The study duration was 6 months from $6^{\text {th }}$ January 2018 to $6^{\text {th }}$ July 2018. Approval was obtained from ethics review committee of the hospital. Patients were informed about the possible complications of FNA procedure and outcome of the study and written, informed consent was taken from each one of them.

Sample size was calculated by taking prevalence of thyroid nodules as $13 \%{ }^{14}$, with $90 \%$ confidence level and $5 \%$ margin of error. Total of 123 patients of either gender, with thyroid nodules detected by ultrasound (GE Logiq P7), classified as TIRADS II-V and scheduled for FNAC, were included. Patients with normal scan of thyroid (TI-RADS class I), already known thyroid pathology and those who refused to be a part of the study, were excluded. Complete clinical examination and required laboratory investigation were done in all patients. All the patients underwent High-resolution B-mode ultrasound scan performed by an experienced radiologist, having more than 5 years of experience. TI-RADS proposed by Horvath et al. in 2009 was followed for classification of thyroid nodules because it has a fair inter-observer agreement. Following ultrasound (USG) evaluation of thyroid gland, USG-guided FNAC was carried out by the same radiologists who performed the USG. A 23gauge needle, linked to a $2 \mathrm{ml}$ disposable plastic syringe and aspirator, were used for FNAC. Aspiration of each lesion was done twice. All the specimens were sent to the pathology laboratory for cytology. The data was recorded on a proforma and analysis was done by using SPSS version 20 . Chi-square test was applied and a $P$-value $<0.05$ was considered as statistically significant. 


\section{Results}

Total 123 patients with thyroid nodules were studied. Mean age of the patients was $45.78 \pm 13.11$ years and the most common age group was 25-40 years (41.5\%). Regarding gender predilection, there were more female patients $(73.2 \%)$ with thyroid nodules than male patients (26.8\%) (Table I).

Table: I. Distribution of study patients according to age groups and gender $(n=123)$

\begin{tabular}{|l|c|}
\hline Variables & $\begin{array}{c}\text { Frequency } \\
\mathbf{n}(\%)\end{array}$ \\
\hline Age groups (years) & $51(41.5 \%)$ \\
\hline $25-40$ & $42(34.1 \%)$ \\
\hline $41-55$ & $30(24.4 \%)$ \\
\hline $56-70$ & 123 \\
\hline Total & $33(26.8 \%)$ \\
\hline Gender & $90(73.2 \%)$ \\
\hline Male & 123 \\
\hline Female & \\
\hline Total &
\end{tabular}

Most of the thyroid nodules detected on USG were classified as TI-RADS class II (56.10\%) (Figure 1).

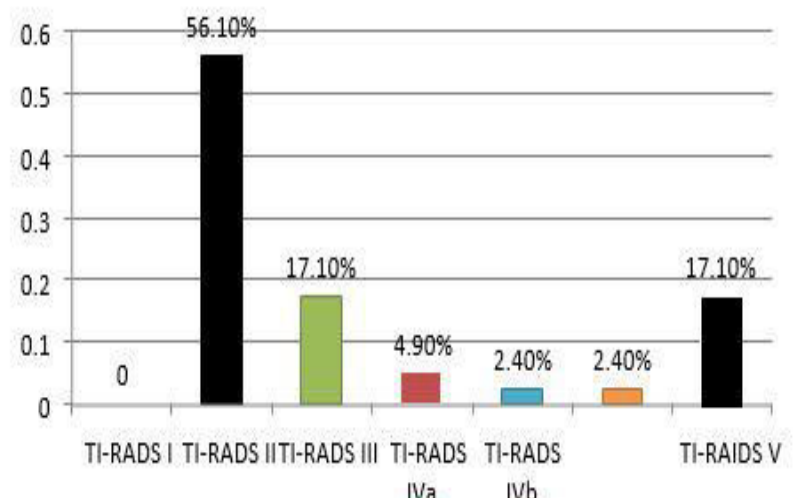

Figure 1: TI-RADS classification of thyroid nodules on USG(n=123)

A significant association was seen between TI-RADS classification of thyroid nodules and findings on cytology. Thyroid nodules with TI-RADS II, III and IVa classification showed benign cytological findings, while TI-RADS class $V$ had a significant association with malignant findings on cytology $(P=0.001)$ (Table II).

\section{Discussion}

The prevalence of thyroid nodules is increasing worldwide. Currently, it is reported to be around $19-67 \%$, of which about $5-15 \%$ are usually malignant. ${ }^{15}$ In Pakistan, the percentage of malignancy is reported to be $11-14.35 \% .{ }^{14}$ In this study, we found a significant association of the TIRAD class $V$ with malignant thyroid nodules on FNAC, while TI-RADS II, III and IVa were significantly associated with benign thyroid nodules.

Our results are comparable to that of Dy and colleagues. ${ }^{13}$ They carried out a retrospective study on 149 patients with thyroid nodules, with majority of them being female patients (87\%). They compared TI-RAD classification with FNAB and concluded that higher TI-RAD class is associated with greater risk of malignancy. They also reported that TI-RADS classification varies between TI-RADS 1 and 5. TI-RADS 1 is compatible with a typical thyroid gland, TI-RADS 2 with benign nodules, TIRADS 3 with possibly benign nodules, TI-RADS 4 with USG characteristics suspicious of malignancy and TI-RADS 5, nodules highly indicative of malignancy. ${ }^{13}$

Results of our study are also in line with the research by Zhuang et al. ${ }^{15}$ In their pursuit to develop a practical TI-RAD system, they also compared the TI-RADS findings with FNAB in 153 patients. They found TI-RADS to be $97 \%$ accurate and the TI-RAD system they devised showed the risk of malignancy in different classes of TIRADS as follows; TIRADS 2(0\% malignancy), TIRADS 3 (3.6\% malignancy), TIRADS 4 (17-75\% malignancy), and TIRADS 5 (98\% malignancy). ${ }^{15}$ 


\begin{tabular}{|c|c|c|c|c|c|c|c|}
\hline \multicolumn{8}{|c|}{ Table II: Association of TI-RADS classification with cytology findings of thyroid nodules ( $\mathrm{n}=123$ ) } \\
\hline \multirow{2}{*}{ TI-RAD classification } & \multicolumn{5}{|c|}{ Cytology findings } & \multirow{2}{*}{ Total } & \multirow{2}{*}{$P$-value* } \\
\hline & Benign & Malignant & $\begin{array}{l}\text { Suspected } \\
\text { malignancy }\end{array}$ & $\begin{array}{c}\text { papillary } \\
\text { carcinoma }\end{array}$ & Cystic fluid & & \\
\hline II & 64 & 0 & 2 & 0 & 3 & 69 & \multirow{7}{*}{0.001} \\
\hline III & 19 & 1 & 2 & 0 & 0 & 22 & \\
\hline IVa & 6 & 0 & 0 & 0 & 0 & 6 & \\
\hline $\mathrm{IVb}$ & 0 & 1 & 1 & 0 & 0 & 2 & \\
\hline IVc & 1 & 1 & 1 & 0 & 0 & 3 & \\
\hline V & 12 & 3 & 3 & 3 & 0 & 21 & \\
\hline Total & 102 & 6 & 9 & 3 & 3 & 123 & \\
\hline
\end{tabular}

$* P$-value $<0.05$ was considered statistically significant

Kwak et al. ${ }^{16}$ utilized a simpler and more practical TI-RADS scoring grounded on BI-RADS category like category 3 (without any suspected features of US), 4a (one suspicious characteristic), 4b (2 suspicious characteristics), 4c (3/4 suspicious characteristics). This facilitated risk stratification of a malignant thyroid nodule. ${ }^{16}$

Vargas-Uricoechea et al. ${ }^{17}$ tried to find concordance of TIRADS with Bethesda cytology criteria. The mean age of patients in their study was 57 years with a predominance of females (75\%). They found the highest concordance between the two modalities in TIRADS class II. They inferred that TIRAD classification of benign nodules usually complements the cytology results therefore unnecessary intervention can be avoided in such patients.

The main limitation of this study was a small sample size and including patients from a single institution, so the results of this study cannot be generalized to the entire population. Second employing FNAB instead of FNAC for comparison with TI-RADS categories would give more reliable results as biopsy is regarded as gold standard for diagnosis of benign and malignant lesions.

\section{Conclusion}

There is a significant association between TI-RAD classification and cytological findings from FNAC of thyroid nodules. TI-RADS classification is a reliable diagnostic modality and can be used for detection of thyroid lesions independent of FNAC, thus reducing the burden of unnecessary biopsies.

\section{References}

1. Nguyen QT, Lee EJ, Huang MG, Park YI, Khullar A, Plodkowski RA. Diagnosis and treatment of patients with thyroid cancer. Am Health Drug Benefits. 2015; 8(1): 30-40. PMID: 25964831

2. Tamhane S, Gharib H. Thyroid nodule update on diagnosis and management. Clin Diabetes Endocrinol. 2016; 2(1): 17. Doi: 10.1186/s40842016-0035-7

3. Shin JH, Baek JH, Chung J, Ha EJ, Kim JH, Lee YH, et al. Ultrasonography diagnosis and imaging-based management of thyroid nodules: revised Korean Society of Thyroid Radiology consensus statement and recommendations. Korean J Radiol. 2016; 17(3): 370-95. Doi: 10.3348/kjr.2016.17.3.370

4. Mandel SJ. A 64-year-old woman with a thyroid nodule. JAMA 2004; 292(21): 2632-42. Doi: 10.1001/jama.292.21.2632

5. Nam-Goong IS, Kim HY, Gong G, Lee HK, Hong SJ, Kim WB, et al. Ultrasonography-guided fine-needle aspiration of thyroid incidentaloma: correlation with pathological findings. Clin Endocrinol (Oxf). 
2004;60(1):21-8. Doi: 10.1046/j.1365-2265.2003.01 912.x

6. Smith-Bindman R, Lebda P, Feldstein VA, Sellami D, Goldstein RB, Brasic N, et al. Risk of thyroid cancer based on thyroid ultrasound imaging characteristics: results of a population-based study. JAMA Intern Med. 2013; 173(19): 1788-96. Doi: 10.1001/ jamainternmed.2013.9245

7. Alshaikh S, Harb Z, Aljufairi E, Almahari SA. Classification of thyroid fine-needle aspiration cytology into Bethesda categories: An institutional experience and review of the literature. CytoJournal. 2018; 15: 4. Doi: 10.4103/cytojournal. cytojournal_32_17

8. Lee YH, Baek JH, Jung SL, Kwak JY, Kim JH, Shin JH, Korean Society of Thyroid Radiology (KSThR). Ultrasound-guided fine needle aspiration of thyroid nodules: a consensus statement by the Korean Society of Thyroid Radiology. Korean J Radiol. 2015; 16(2): 391-401. Doi: 10.3348/kjr.2015.16.2.391.

9. Basharat $\mathrm{R}$, Bukhari $\mathrm{MH}$, Saeed $\mathrm{S}$, Hamid $\mathrm{T}$. Comparison of fine needle aspiration cytology and thyroid scan in solitary thyroid nodule. Patholog Res Int. 2011; 2011: 754041. Doi: 10.4061/2011/754041.

10. Songsaeng D. Siriraj thyroid imaging reporting and data system and its efficacy. Siriraj Med J. 2017; 69(5): 262-7. Doi: 10.14456/smj.2017.52

11. Horvath E, Majlis S, Rossi R, Franco C, Niedmann JP, Castro $A$, et al. An ultrasonogram reporting system for thyroid nodules stratifying cancer risk for clinical management. J Clin Endocrinol Metab. 2009; 94(5): 1748-51. Doi: 10.1210/jc.2008-1724
12. Ashamallah GA, EL-Adalany MA. Risk for malignancy of thyroid nodules: Comparative study between TIRADS and US based classification system. Egypt J Radiol Nucl Med. 2016; 47(4): 1373-84. Doi: 10.1016/j.ejrnm.2016.08.021

13. Dy JG, Kasala R, Yao C, Ongoco R, Mojica DJ. Thyroid Imaging Reporting and Data System (TIRADS) in Stratifying Risk of Thyroid Malignancy at The Medical City. J ASEAN Fed Endocr Soc 2017; 32(2): 108

14. Kamran M, Hassan N, Ali M, Ahmad F, Shahzad S, Zehra N. Frequency of thyroid incidentalomas in Karachi population. Pak J Med Sci. 2014; 30(4): 79397. Doi: $10.12669 /$ pjms.304.4808

15. Zhuang Y, Li C, Hua Z, Chen K, Lin JL. A novel TIRADS of US classification. Biomedical engineering online. 2018; 17(1): 82. Doi: 10.15605/jafes.032.02.03

16. Kwak JY, Kim HH, Yoon, JH, Moon HJ, Son EJ, et al. Thyroid imaging reporting and data system for US features of nodules: A step in establishing better stratification of cancer risk. Radiology. 2011; 260(3): 892-9. Doi: 10.1148/radiol.11110206

17. Vargas-Uricoechea H, Meza-Cabrera I, HerreraChaparro J. Concordance between the TIRADS ultrasound criteria and the BETHESDA cytology criteria on the nontoxic thyroid nodule. Thyroid Res. 2017; 10(1): 1. Doi: 10.1186/s13044-017-0037-2 